\title{
Improvement of bovine in vitro embryo production by vitamin $K_{2}$ supplementation
}

\author{
Luis Manuel Baldoceda-Baldeon, Dominic Gagné, Christian Vigneault ${ }^{1}$, Patrick Blondin ${ }^{1}$ and \\ Claude Robert \\ Laboratory of Functional Genomics of Early Embryonic Development, Faculté des Sciences de L'Agriculture et de \\ L'Alimentation, Pavillon des Services, Institut des Nutraceutiques et des Aliments Fonctionnels (INAF), Centre de \\ Recherche en Biologie de la Reproduction, Université Laval, Local 2742, Quebec, Canada G1V OA6 and \\ ${ }^{1}$ L'Alliance Boviteq, Inc., 19320 Grand Rang St-François, Saint-Hyacinthe, Quebec, Canada J2T 5H1
}

Correspondence should be addressed to C Robert; Email: claude.robert@fsaa.ulaval.ca

\begin{abstract}
Mitochondria play an important role during early development in mammalian embryos. It has been shown that properly controlled follicular preparation increases the likelihood of in-vitro-produced bovine embryos reaching the blastocyst stage and that competent embryos exhibit heightened expression of genes associated with mitochondrial function. We hypothesized that apparently incompetent embryos could be rescued by restoring mitochondrial function. It has been shown that vitamin $\mathrm{K}_{2}$ (a membrane-bound electron carrier similar to ubiquinone) can restore mitochondrial dysfunction in eukaryotic cells. The aim of this study was therefore to investigate the effects of vitamin $\mathrm{K}_{2}$ on bovine embryonic development in vitro. The vitamin was found most effective when added $72 \mathrm{~h}$ after fertilization. It produced a significant $(P<0.05)$ increase in the percentage of blastocysts $(+8.6 \%)$, more expanded blastocysts $(+7.8 \%)$, and embryos of better morphological quality. It improved the mitochondrial activity significantly and had a measurable impact on gene expression. This is the first demonstration that current standard conditions of in vitro production of bovine embryos may be inadequate due to the lack of support for mitochondrial function and may be improved significantly by supplementing the culture medium with vitamin $K_{2}$.

Reproduction (2014) 148 489-497
\end{abstract}

\section{Introduction}

Mitochondria are intracellular organelles that ensure a multitude of key cellular functions such as energy production, control of apoptosis, lipid metabolism, steroid synthesis, and production of metabolites having important regulatory roles in various signaling pathways (Dumollard et al. 2009, Van Blerkom 2009). Although mitochondria are involved in several vital functions, it is their role in balancing oxidative stress and producing ATP that have been most investigated (Thompson et al. 2000, Trimarchi et al. 2000, Dumollard et al. 2009, Van Blerkom 2009). An atypical mitochondrial contingent composed of immature organelles having limited cristae and hence a limited potential for ATP production via oxidative phosphorylation and glycolysis has been observed in oocytes (Trimarchi et al. 2000, Fair et al. 2001, Crocco et al. 2011). This lower level of energy production lasts apparently until these organelles undergo a transition to mitochondria of mature form, which coincides with the activation of the embryonic genome (Thompson et al. 1996, Tarazona et al. 2006, Dumollard et al. 2009). Culture media produce adverse effects on the structure and function of mitochondria, with consequences for the quality of embryos produced in vitro (Crosier et al. 2001, Abe et al. 2002a). The expression of genes associated with embryo viability may be modified (Rizos et al. 2003, Plourde et al. 2012). However, variations in mitochondrial function in association with immature structure have also been observed in embryos produced in vivo (Crosier et al. 2001, Fair et al. 2001, Abe et al. 2002a,b). Meanwhile, oxidative stress has been shown to perturb mitochondrial function leading to a marked reduction in the embryonic development (Bavister 1995, Trimarchi et al. 2000, Van Blerkom 2004). This problem has been studied extensively, and several modifications to culture media (primarily the addition of antioxidants) have been proposed (Olson \& Seidel 2000, Sudano et al. 2010). However, this approach has brought only marginal improvements in embryo competence thus far.

Vitamin $K_{2}$ has been used recently to counteract the deleterious effects on mitochondrial function in a modified strain of Drosophila mutated for a gene that is known to be involved in Parkinson's disease (Vos et al. 2012). It was thus found that vitamin $K_{2}$ played an electron carrier role in the mitochondrial electron 
transport chain complex, resulting in more efficient oxygen use and production of ATP.

Based on our previous study (Plourde et al. 2012), we hypothesized that vitamin $\mathrm{K}_{2}$ could rescue dysfunctional mitochondria, which would translate into a higher percentage of embryos reaching the blastocyst stage. The objectives of this study were to characterize, for first time, the effects of vitamin $K_{2}$ on bovine embryo development and mitochondrial function.

\section{Materials and methods}

All chemicals were purchased from Sigma-Aldrich unless specified otherwise.

\section{Recovery of oocytes for embryo production in vitro}

Ovaries removed immediately from cows slaughtered in a commercial abattoir were transported in saline $(0.9 \% \mathrm{NaCl})$ containing $1 \%$ antifungal agent. Oocytes were collected and matured, followed by fertilization and culture according to standard in vitro techniques in our laboratory (Plourde et al. 2012). Cumulus-oocyte complex (COC) was retrieved from follicles 2-6 mm in diameter using a $38 \mathrm{~mm} 18 \mathrm{G}$ needle attached to a 10-ml syringe. Homogeneous COCs with at least five layers of cumulus surrounding the oocyte completely were selected, while COCs exhibiting fragmented cytoplasm, pyknotic cumulus, pale nuclei, or abnormal morphology were automatically rejected.

\section{Maturation of oocytes}

COCs were placed in HEPES-buffered Tyrode's lactate medium (TLH) supplemented with $10 \%$ bovine serum, $200 \mu$ M pyruvate, and $50 \mu \mathrm{g} / \mathrm{ml}$ gentamycin and washed twice thoroughly to ensure total removal of follicular liquid. Groups of ten healthy COCs were placed in $50-\mu \mathrm{l}$ droplets of the medium under $9 \mathrm{ml}$ of filtered mineral oil (\#8410, Sigma). The maturation medium was composed of TCM199 (Gibco product 11150-059, Invitrogen) plus $10 \%$ FCS (Sterile Foetal Bovine Serum for Cell Culture, Medicorp, Montreal, QC, Canada), $200 \mu \mathrm{M}$ pyruvate, $50 \mu \mathrm{g} / \mathrm{ml}$ gentamycin, and $0.1 \mu \mathrm{g} / \mathrm{ml}$ follicle-stimulating hormone (Gonal-f, Serono Canada, Inc.). Droplets containing COCs were incubated for $24 \mathrm{~h}$ at $38.5^{\circ} \mathrm{C}$ in a humidified (100\%) atmosphere containing $5 \% \mathrm{CO}_{2}$ and $20 \% \mathrm{O}_{2}$.

\section{Fertilization of oocytes}

Mature COCs were washed twice in TLH. Groups of five mature COCs were added to $50-\mu \mathrm{l}$ droplets of TLH supplemented with $0.6 \%$ BSA (Sigma fraction V) and $200 \mu \mathrm{M}$ gentamycin (IVF medium) and covered with filtered mineral oil. Two microliter of solution containing $1 \mathrm{mM}$ hypotaurine, $2 \mathrm{mM}$ penicillamine, and $250 \mathrm{mM}$ epinephrine were then added to the COC-containing droplets. Oocytes were fertilized with frozen semen after $15-18 \mathrm{~h}$ of incubation at $38.5^{\circ} \mathrm{C}$ in a humidified atmosphere containing $5 \% \mathrm{CO}_{2}$ and $20 \% \mathrm{O}_{2}$. Cryopreserved pooled bull ejaculate was thawed at $37^{\circ} \mathrm{C}$ in a water bath, laid on a discontinuous Percoll gradient $(2 \mathrm{ml}$ of $45 \%$ Percoll over $2 \mathrm{ml}$ of $90 \%$ Percoll) and centrifuged at $700 \mathrm{~g}$ for $30 \mathrm{~min}$ at room temperature. The supernatant was discarded and the pellet was re-suspended in the in vitro fertilization medium to obtain a concentration of 10000 spermatozoa per COC (five COCs per droplet) based on counts using a hemocytometer.

\section{Culture of embryos}

Presumptive zygotes were denuded of cumulus cells and spermatozoa by gentle pipetting, washed three times in TLH supplemented with fatty-acid-free BSA, and then cultured in three steps in a synthetic oviduct fluid (SOF) containing essential and non-essential amino acids, $0.5 \mathrm{mM}$ of glycyl-glutamine and $0.4 \%$ fatty-acid-free BSA (ICP-Bio, Auckland, New Zealand) under filtered mineral oil at $38.5^{\circ} \mathrm{C}$ in a humidified atmosphere containing $6.5 \% \mathrm{CO}_{2}, 5 \% \mathrm{O}_{2}$, and $88.5 \% \mathrm{~N}_{2}$. Embryos were placed in groups of ten in droplets of SOF $(10 \mu \mathrm{l})$. Embryonic culture was following three media change. For the first change, the SOF contained only non-essential amino acids at the standard concentration plus $3 \mu \mathrm{M}$ EDTA. For the second change (72 h post-fertilization), the SOF also contained essential amino acids at half this concentration (SOF2). For the third change (120 $\mathrm{h}$ post-fertilization), both the non-essential and essential amino acids were present at the standard concentration (SOF3).

Vitamin $K_{2}$ was added in accordance with Vos et al. (2012) with some modifications. Briefly, vitamin $\mathrm{K}_{2}$ dissolved in $95 \%$ ethanol solution (HPLC grade, Fisher Scientific, Fair Lawn, NJ, USA) and stored in the dark at $-20{ }^{\circ} \mathrm{C}$ as a $400 \times$ stock solution was diluted in SOF to obtain final concentrations of $0.5 \mathrm{mM}$ vitamin $\mathrm{K}_{2}$ and $0.25 \%$ ethanol.

At $72 \mathrm{~h}$ post-fertilization, embryos were transferred randomly to droplets $(10 \mu \mathrm{l})$ of SOF2 containing added vitamin $\mathrm{K}_{2}$ or not. The vitamin-supplemented and control groups were thus both supplemented with $0.25 \%$ ethanol (previous tests indicated that $0.25 \%$ ethanol had no effect on bovine embryo development in vitro). Embryos were transferred at $120 \mathrm{~h}$ post-fertilization to $20 \mu \mathrm{l}$ droplets of SOF3 again containing added vitamin $\mathrm{K}_{2}$ or not (with $0.25 \%$ ethanol in either case). This medium was replaced three times to prevent toxicity due to ammonium accumulation and nutrient (amino acid) depletion due to assimilation. Embryos remained under these conditions until day 7 , when blastocyst percentages were recorded and early, expanded, and hatched blastocysts were categorized according to the IETS system and used in the various tests in this study. Embryos were either processed fresh or collected, snap-frozen in liquid nitrogen, and stored at $-80{ }^{\circ} \mathrm{C}$ until use.

\section{Characterization of embryo mitochondrial activity}

Under an atmosphere containing $5 \% \mathrm{CO}_{2}$ at $38^{\circ} \mathrm{C}$, live embryos in SOF were stained for $40 \mathrm{~min}$ with the active mitochondrial dye CMX-rosamine (Mitotracker Red, Molecular Probes, Eugene, OR, USA) at a concentration of $300 \mathrm{nM}$ (same atmosphere and temperature). This dye has been found to be more sensitive to the mitochondrial membrane potential and mitochondrial protein (thiol group abundance) than other dyes and to exhibit better retention in the organelle and more homogenous dispersion throughout all embryonic cells 
(Poot et al. 1996). The fluorescence excitation wavelength was $594 \mathrm{~nm}$ and emission was read at $608 \mathrm{~nm}$. Carbonyl cyanide m-chlorophenylhydrazone (CCCP), which uncouples mitochondrial membrane potential, was used at a concentration of $100 \mathrm{nM}$ in order to provide a negative control. The CCСP treatment provided background values against which the different measurement runs were calibrated. Embryos were selected randomly for treatment with $\mathrm{CMX}$-rosamine or with $\mathrm{CCCP}+\mathrm{CMX}$-rosamine.

\section{Confocal microscopy}

Confocal microscopy was performed using a Nikon TE2000 confocal microscope (Nikon, Mississauga, ON, Canada) with a water-immersion objective at an optical magnification of 60 . Embryo morphological phenotype was recorded in photos in gray scale taken with the same settings to estimate color (dark or pale) based on the IETS system. Mitochondrial activity was recorded as an epifluorescent image of $\mathrm{CMX}$-rosamine dye in gray scale.

Digital images of embryos stained with CMX-rosamine were obtained using a LSM 700 confocal system with a Zeiss Axiovert $200 \mathrm{M}$ inverted microscope (Carl Zeiss $\mathrm{GmbH}$, Jena, Germany). The embryos were viewed with the PlanApochromat $40 \times$ lens $(\mathrm{NA}=1.2)$ at $10 \%$ of the utilizable laser intensity (maximum power: $1.2 \mathrm{~W}$, output: $25 \%$ of the maximum tube current) and an HFT dichroic beam splitter $(458 / 514 \mathrm{~nm})$. Images of orthogonal projections consisting of 21 slices ( $1 \mu \mathrm{m}$ each) were acquired as 'lambda stacks' using the Lambda Mode scanning procedure at a resolution of $1024 \times 1024$ pixels. The microscope settings and the lambda mode scanning procedure were the same for all collected lambda stacks. The intensity of CMX-rosamine fluorescence was measured using the mean gray scale in the freely available Image J Software (Abramoff et al. 2004). Results are expressed in mean fluorescence intensity arbitrary units (AUs) based on all samples within a group.

\section{Extraction of total DNA and RNA}

Additional blastocysts from each group were used to obtain values for total genomic DNA and RNA. The nucleic acids were extracted simultaneously using the AllPrep DNA/RNA Micro Kit (Qiagen) according to the manufacturer's instructions. Genomic DNA was used in the mitochondrial DNA
(mtDNA) quantification procedure described below and total RNA was reverse transcribed for quantification by RT-PCR.

\section{Quantification of $m t D N A$}

mtDNA in individual embryos $(n=10$ biological replicates per treatment) was quantified using a PCR (qPCR) method. The $12 S$ rRNA gene (GenBank accession number: J01394) was selected as a mitochondrial target and the $M X 1$ gene (GenBank accession number: AY340484) as a nuclear target (Table 1). The mtDNA and nuclear DNA (nDNA) were used to calculate the relative concentration of mtDNA in each embryo, which was expressed as the mtDNA/nDNA ratio. The LightCycler 2.0 (Roche Diagnostics) was used to monitor qPCRs. The reaction mixture $(20 \mu \mathrm{l})$ contained $0.5 \mu \mathrm{l}$ of each primer solution $(0.25 \mu \mathrm{M}), 1.2 \mu \mathrm{l}$ of $1.5 \mu \mathrm{M} \mathrm{MgCl}_{2}, 2 \mu \mathrm{l}$ of LightCycler FastStart DNA Master SYBR Green I (Roche Diagnostics), and $5 \mu \mathrm{l}$ of DNA sample. The average DNA concentration of each sample was $0.00335 \mathrm{ng} / \mu \mathrm{l}$. The following cycling conditions were applied for amplification: initial denaturation at $95^{\circ} \mathrm{C}$ for 10 min followed by 50 cycles of $95^{\circ} \mathrm{C}$ for $5 \mathrm{~s}, 5 \mathrm{~s}$ ( $12 \mathrm{~S}$ rRNA) at 58 or $60{ }^{\circ} \mathrm{C}(M X 1)$, followed by $72{ }^{\circ} \mathrm{C}$ for $20 \mathrm{~s}$ and $76{ }^{\circ} \mathrm{C}$ (12S rRNA) or $85^{\circ} \mathrm{C}(\mathrm{MX} 1)$ for $5 \mathrm{~s}$. The presence of amplicons was verified using melting curve analysis: Following the last amplification cycle, the internal temperature of the LightCycler was increased rapidly to $94{ }^{\circ} \mathrm{C}$ then decreased to $72{ }^{\circ} \mathrm{C}$ for $30 \mathrm{~s}$, followed by an increase to $94{ }^{\circ} \mathrm{C}$ at a rate of $0.1{ }^{\circ} \mathrm{C} / \mathrm{s}$, with continuous fluorescence reading. Copy numbers of mtDNA and nDNA were obtained from a standard curve, which was based on the linear relationship between the crossing point cycle values and the logarithm of the starting copy number.

\section{Determination of differential gene expression in blastocysts}

Total RNA extracted from embryos ( $n=4$ biological replicates for each group) was purified using the PicoPure RNA Kit (Molecular Devices, Downingtown, PA, USA) according to the manufacturer's instructions. DNA was digested with DNase I (Qiagen). The RNA concentration and quality were measured using the 2100-Bioanalyzer (Agilent Technologies, Palo Alto, CA, USA) with the RNA PicoLab Chip (Agilent Technologies). Only RNA of very good quality (RIN over 8) was used for amplification.

Table 1 Characteristics of the primers used for gene candidate transcript quantification.

\begin{tabular}{|c|c|c|c|c|c|c|}
\hline \multirow[b]{2}{*}{ Symbols } & \multirow{2}{*}{$\begin{array}{l}\text { Accession } \\
\text { numbers }\end{array}$} & \multicolumn{2}{|c|}{ Primer sequences } & \multirow{2}{*}{$\begin{array}{c}\text { Annealing } \\
\text { temperature } \\
\left({ }^{\circ} \mathrm{C}\right)\end{array}$} & \multirow{2}{*}{$\begin{array}{c}\text { Acquisition } \\
\text { temperature } \\
\left({ }^{\circ} \mathrm{C}\right)\end{array}$} & \multirow{2}{*}{$\begin{array}{l}\text { Product } \\
\text { size }(b p) \\
\end{array}$} \\
\hline & & Fw $\left(5^{\prime}-3^{\prime}\right)$ & $\operatorname{Rv}\left(5^{\prime}-3^{\prime}\right)$ & & & \\
\hline$A C A A 2$ & NM_001035342 & TGGTTTCCAGTCCATTGTGA & TTCСАССТСТGСТGTGATTG & 57 & 81 & 291 \\
\hline ATP2B4 & NM_001172594 & ACGGCTGTGGGTATCAACTC & TCAGACCAGCTTTCCCAATC & 57 & 85 & 256 \\
\hline NDUFST & NM_001038022 & GTCCGACGTGATGATTGTGG & CCCGTTTGATCTTCTTCTGC & 57 & 89 & 263 \\
\hline CERS2 & NM_001034667 & CACCCAGTTGCСTTTAAACC & CCACAGGTGGGACAGTAAGG & 57 & 82 & 254 \\
\hline MRPST & NM_001034343 & TGAGTGCAGGGAGAAGAAGC & CTGGCCTTAAGAGGGAACG & 57 & 89 & 256 \\
\hline$M \times 1$ & AY_ $\_-340484$ & $\begin{array}{l}\text { ATGCGTGCTATTGGCTCT- } \\
\text { TCCTCA }\end{array}$ & $\begin{array}{l}\text { CAAACAGAGCAAGG- } \\
\text { GAGTTTGGCA }\end{array}$ & 60 & 85 & 181 \\
\hline $12 S$ rRNA & J0_1394 & TCGATAAACCCCGATAAACC & TTCGTGCTTGATTCTCTTGG & 58 & 76 & 186 \\
\hline
\end{tabular}


The concentration of purified RNA amplified for two rounds (with T7 RNA polymerase) using the RiboAmp HSPlus RNA Amplification Kit (Life Science, Foster City, CA, USA) was measured using a NanoDrop ND-1000 spectrophotometer (NanoDrop, Wilmington, DE, USA). Antisense RNA labeled with Cy3 or Cy5 using the Universal Linkage System Kit (Kreatech Diagnostic, Amsterdam, The Netherlands) was hybridized (825 ng) on Agilent-manufactured EmbryoGENE slides (Robert et al. 2011) in a two-color dye-swap design in a hybridization oven for $17 \mathrm{~h}$ at $65^{\circ} \mathrm{C}$. A simple direct comparison of the treatments was made using four independent biological replicates (each consisting of one blastocyst) per treatment. Microarray slides were then washed and scanned with the PowerScanner (Tecan, Männedorf, Switzerland) and analyzed using the Array-Pro Analyzer Software (MediaCybernetics, Bethesda, MD, USA).

Microarray data were pre-processed as described in previous studies (Plourde et al. 2012). Briefly, data intensity files were analyzed using FlexArray 1.6.1 (http://genomequebec.mcgill. ca/FlexArray), in which raw data corrected by background subtraction were pre-processed by performing a Lowess intra-array and Quantile inter-array normalizations. Statistically significant variations were detected using Limma (Bioconductor, hosting provided by Fred Hutchinson Cancer Research Center, Seattle, WA, USA). Gene expression differentials were considered significant when they were at least 1.2 and the cut-off adjusted $P$ value was $<0.01$. Pathway analyses and downstream exploitation of gene lists were carried out using Ingenuity Pathway Analysis Software Version 8.6 (Ingenuity Systems, Inc., Redwood City, CA, USA).

\section{Validation of $m R$ RA levels by quantitative RT-PCR}

Microarray results were validated using single embryos $(n=5$ biological replications per group). Total RNA (extracted as described above) was reverse transcribed using the qScript cDNA SuperMix (Quanta Biosciences, Gaithersburg, MD, USA) with oligo-dT to prime the reaction as per the manufacturer's recommendations. Primers of candidates (acetyl-CoA acyltransferase 2 or $A C A A 2, \mathrm{Ca}^{2+}$ transporting plasma membrane 4 ATPase or ATP2B4, NADH dehydrogenase (ubiquinone) Fe-S protein 7 or NDUFS7, $20 \mathrm{kDa}$ NADH-coenzyme Q reductase, ceramide synthase 2 or CERS2, and mitochondrial ribosomal protein S7 or MRPS7) were designed using the Primer3 Web interface (http://frodo.wi.mit.edu/cgi-bin/primer3/primer3_ www.cgi) and synthetized at IDT (Coralville, IA, USA). The reaction mixture was composed of the LightCycler FastStart DNA Master SYBR Green I Kit components (Roche Diagnostics) and real-time measurements were performed in a LightCycler 2.0 apparatus (Roche Diagnostics). GenBank accession numbers, primer sequences, annealing temperatures, and product sizes are given in Table 1.

For quantification, real-time PCR was performed as described previously (Bermejo-Alvarez et al. 2010). The comparative cycle threshold $(\Delta C T)$ method was then used to quantify expression levels, as described by Schmittgen \& Livak (2008). Quantification was normalized to the endogenous control ( $\beta$-actin to account for cell numbers). A change in the relative level of gene expression of the target was calculated as $2-\Delta \Delta C \mathrm{C}$.

\section{Electron microscopy}

Early blastocysts ( $n=5$ biological replicates for both treatments) classified as good quality were washed three times in PBS containing $10 \%$ FCS at $4{ }^{\circ} \mathrm{C}$. Embryos were fixed in freshly prepared $3 \%$ glutaraldehyde (Electron Microscope Solutions, Ft. Washington, PA, USA) in $0.1 \mathrm{M}$ PBS for $1 \mathrm{~h}$ at $4{ }^{\circ} \mathrm{C}$, stored in $0.1 \mathrm{MPBS}$ and later embedded individually at $37{ }^{\circ} \mathrm{C}$ in $4 \%(\mathrm{w} / \mathrm{v})$ agarose (low melting temperature, prepared at $\sim 55^{\circ} \mathrm{C}$ ), and isolated as blocks using a razor blade and a stereomicroscope. The blocks were washed twice in PBS for $5 \mathrm{~min}$ at room temperature, post-fixed for $1 \mathrm{~h}$ at room temperature in $4 \%$ osmium tetroxide $\left(\mathrm{OsO}_{4}\right)$ in PBS, dehydrated gradually in a series of acetone solutions, and then embedded in Epon 812 epoxy resin. Sections of $1 \mu \mathrm{m}$ thickness were sliced through the entire embryo using a glass knife on a Reichert Jung Ultracut E ultramicrotome and stained with toluidine blue. Sections of 80-100 nm thickness were sliced with a diamond knife and collected on Formvar-carbon-coated 200 mesh nickel grids. Sections were then stained with uranyl acetate and lead citrate and examined using a FEI Tecnai 12 (Eindhoven, The Netherlands) transmission electron microscope operated at $80 \mathrm{kV}$. Observations were made on four different sections per embryo.

\section{Statistical analysis}

Samples were distributed randomly among the vitamin-treated and control groups. The blastocyst rate (percentage) was calculated from the total number of oocytes selected for maturation and data were analyzed using a paired $t$-test. The mitochondrial activity based on Mitotracker Red intensity was analyzed using Student's t-test to identify significant differences between groups. All tests were performed using the Prism Version 5.0 statistical software package (GraphPad Software, La Jolla, CA, USA). Differences between groups were declared significant when $P<0.05$.

\section{Results}

\section{Effects of vitamin $K_{2}$ on bovine embryo development in vitro}

Timing of vitamin $K_{2}$ supplementation of the culture medium was based on preliminary experiments that showed detrimental effects when the vitamin was added during oocyte maturation or early post-fertilization stages (data not shown). It was noted that vitamin $K_{2}$ supplementation was effective from the eight-cell stage onward. All measurements were carried out on embryos treated at $72 \mathrm{~h}$ post-fertilization.

Supplementation of the culture medium with vitamin $\mathrm{K}_{2}$ increased the percentage of blastocysts by $8.6 \%$. The overall appearance of the blastocysts is shown in Fig. 1. Visible differences were few and trivial (e.g. extruded blastomeres) and were observed more frequently in the control group. On day 7, the percentage of expanded blastocysts (Fig. 2) was significantly higher in the vitamin-treated group $(17.19 \%)$ than in the control group $(10.0 \%)$. The quality of the embryos in the 


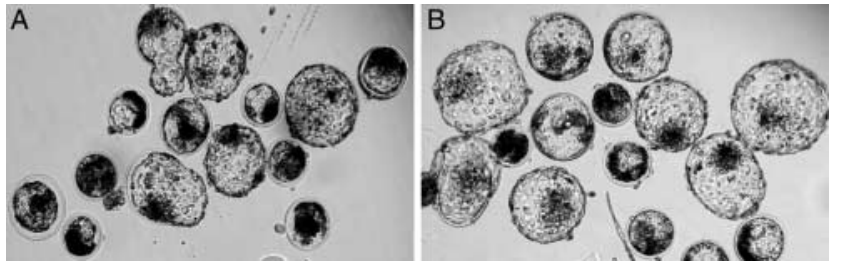

Figure 1 Bovine embryos produced in vitro and matured for 7 days in a synthetic oviduct fluid, (A) control group and (B) treated with vitamin $\mathrm{K}_{2}$. Images were taken at $40 \times$ magnification.

vitamin-treated group may be classified as 'excellent' compared with the control group, based on IETS criteria.

\section{Blastocyst rate and mitochondrial activity are both increased in embryos treated with vitamin $K_{2}$}

Figure $3 \mathrm{~A}$ and $\mathrm{B}$ shows the distribution of active mitochondria (red) in bovine embryos obtained in vitro, as observed using confocal microscopy. As expected, mitochondrial activity (based on Mitotracker fluorescence intensity, Fig. 3C) was greater in embryos cultured in the vitamin- $\mathrm{K}_{2}$-supplemented medium $(12603 \pm 88 \mathrm{AU})$ than in control embryos (8960 \pm $92 \mathrm{AU})$. The mtDNA contents normalized on a per-cell basis (Rao et al. 2009) did not differ, suggesting that the mitochondria were more active and not simply greater in number (Fig. 3D).

\section{Vitamin $K_{2}$ supplementation of the early embryo in vitro culture medium influences gene expression at the blastocyst stage}

Of the 37238 gene transcripts surveyed in the bovine in vitro embryos using the microarray, only 37 showed a significant $(P<0.01)$ change at the expression level (by a factor of at least 1.2) in association with the addition of vitamin $\mathrm{K}_{2}$ to the culture medium.

Enrichment analysis showed that the cellular functions with the most significant values were calcium transport and $\beta$-oxidation of fatty acids. These suggest that vitamin $\mathrm{K}_{2}$ had an impact on mitochondrial functions. The microarray results were validated by qRT-PCR analysis using five candidate genes (ACAA2, ATP2B4, NDUFS7, CERS2, and MRPS7) associated with mitochondrial functions (Table 1). The increased expression of ACAA2 in the vitamin $\mathrm{K}_{2}$ group based on hybridization results was thus confirmed (Fig. 4). In the case of the other genes, qRT-PCR analysis tended to confirm the increased expression, albeit with a lesser degree of certainty.

\section{Effect of vitamin $K_{2}$ on mitochondrial morphology in bovine embryos in vitro}

The potential impact of vitamin $\mathrm{K}_{2}$ on mitochondrial maturation was investigated using electron microscopy. Mitochondria of different morphological types in bovine blastomeres have been reported in several studies (Crosier et al. 2001, Fair et al. 2001, Abe et al. 2002a,b, Crocco et al. 2011, 2013). The two most frequently noted types are known as 'mature' (i.e. round or elongated with transverse cristae distributed either in only part of or in the whole organelle) and 'immature' (i.e. rounded and swollen forms in which the matrix is less dense, cristae area is small and retracted toward the periphery or absent, and small vacuoles may be present).

Both of these mitochondrial types were present in blastocysts obtained by culture in both the medium supplemented with vitamin $\mathrm{K}_{2}$ and the control medium. We observed a larger proportion of mitochondria of swollen shape in embryos in the control group (Fig. 5A and $\mathrm{C}$ ), while the blastomeres of embryos cultured in medium with added vitamin $\mathrm{K}_{2}$ contained more mitochondria of the mature shape (Fig. 5B and D).

\section{Discussion}

Mitochondria are vital organelles that play crucial roles in eukaryotic cells not only by generating metabolic energy and managing lipid reserves but also by controlling apoptotic equilibrium and thereby determining cell fate (Dumollard et al. 2009, Van Blerkom 2009). Mitochondrial disorders can lead to the arrest of early development (Bavister 1995, Trimarchi et al. 2000, Van Blerkom 2009). In cattle embryos, effects of the suboptimal in vitro microenvironment on mitochondria have been shown to decrease developmental competence (Crosier et al. 2001, Fair et al. 2001, Abe et al. 2002a,b, Crocco et al. 2011, 2013). We have observed that developmental competence during in vitro production of embryos is improved to $55 \%$ for immature oocytes collected by transvaginal pick-up (OPU) following a dedicated ovarian stimulation regimen, compared with $35 \%$ for those collected from ovaries post-mortem (Plourde et al. 2012). Analysis of the transcriptome showed that the resulting early blastocysts

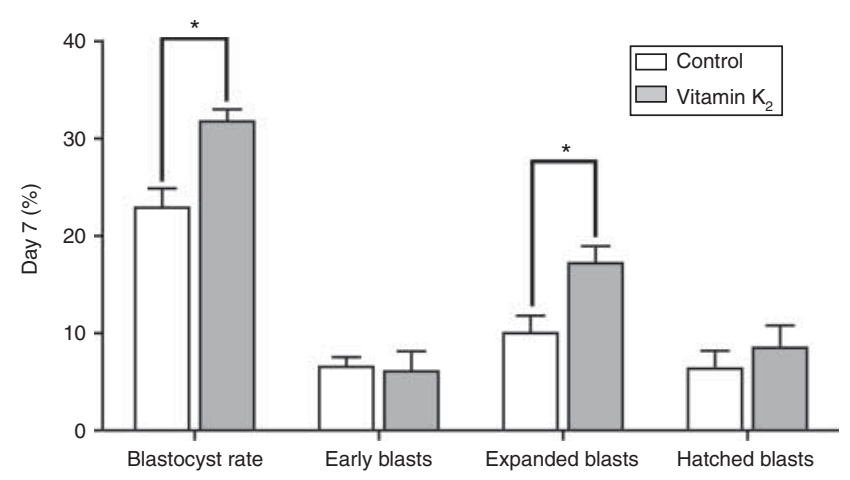

Figure 2 Effect of vitamin $\mathrm{K}_{2}$ on bovine embryo development in vitro. Embryos were cultured in a synthetic oviduct fluid without (white) or with vitamin $\mathrm{K}_{2}$ (gray). Values are expressed as mean \pm s.E.M.

*Significantly different $(P<0.05)$. 

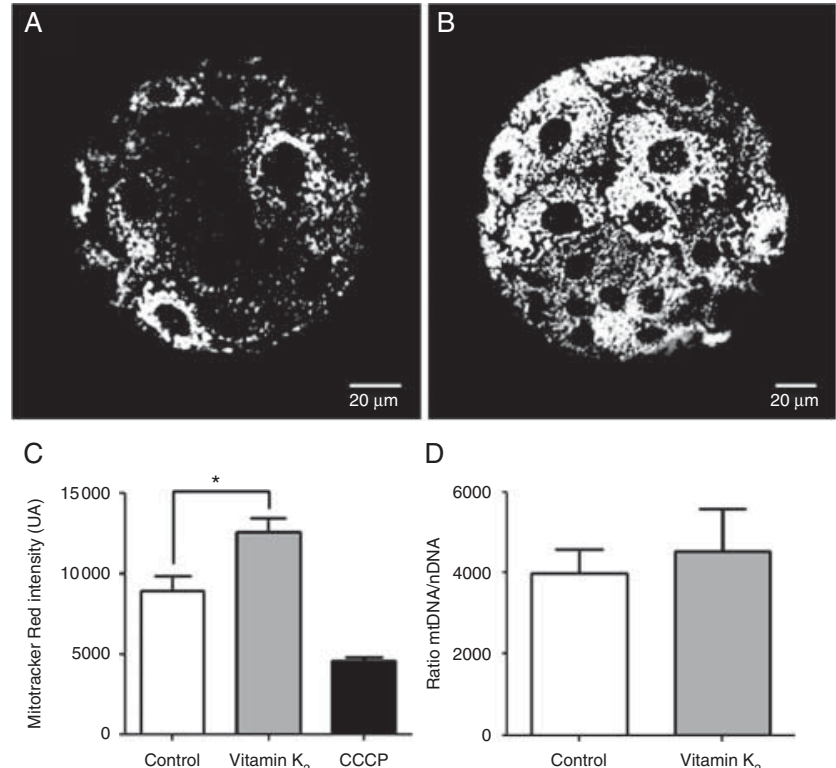

Figure 3 Confocal microscopic images of bovine blastocyst-stage embryos obtained in vitro and stained with Mitotracker Red (CMX-rosamine) to show active mitochondria: (A) embryo cultured in a synthetic oviduct fluid (SOF), (B) embryo cultured in a SOF enriched with vitamin $\mathrm{K}_{2} 72 \mathrm{~h}$ after fertilization, (C) Mitotracker Red fluorescence intensity (carbonyl cyanide m-chlorophenylhydrazone (CCCP), used as a negative control), and (D) ratio of mitochondrial DNA to nuclear DNA in single blastocysts. Values are expressed as mean \pm S.E.M. $*$ Significantly different $(P<0.05)$. AU, arbitrary unit.

were very similar regardless of the oocyte collection method, except for the expression levels of genes associated with mitochondrial functions, which were not affected when the oocytes were collected by OPU from stimulated ovaries. It is well accepted that developmental competence is influenced greatly by the quality of the oocyte (Rizos et al. 2002). We hypothesized that this maternal preparation is associated in part with the state of readiness of the mitochondrial contingent found in the oocyte and that providing support for mitochondrial functions might compensate for suboptimal preparation and thus result in higher developmental competence.

The possibility that the impaired mitochondrial functions observed in embryos developed under in vitro conditions could be due to increased free radical stress has been investigated in several studies. Reducing oxygen tension and adding antioxidants such as vitamins $\mathrm{C}$ and $\mathrm{E}$ to the culture medium have been investigated as means of reducing the free radical load (Olson \& Seidel 2000, Sudano et al. 2010) and reducing free radical generation associated with lipid catabolism (Phongnimitr et al. 2013, Takahashi et al. 2013). The antioxidant approach thus far has brought only marginal improvements in developmental competence. This indicates either that free radicals are not the source of the problem or that the ability of mitochondria to fulfill their multifaceted role is compromised even in the presence of lower levels of free radicals.

In this study, we tested the effect of vitamin $K_{2}$ on bovine embryonic development in vitro. Vitamin $\mathrm{K}_{2}$ has been shown recently to restore the mitochondrial function by acting as an electron carrier (Vos et al. 2012). We have shown herein that adding vitamin $K_{2}$ to the culture medium $72 \mathrm{~h}$ after fertilization significantly increased the percentage of embryos that reach the blastocyst stage. Preliminary tests suggested that adding the vitamin earlier does not have this effect and may even be detrimental. The timing of the supplementation thus coincides with the slight increase in mitochondrial activity observed at the morula stage (72-168 $\mathrm{h}$ after fertilization), which happens when activation of the embryonic genome occurs in cattle (Thompson et al. 1996, Tarazona et al. 2006, Crocco et al. 2011). It is known that this transition is critical for bovine early development, as most developmental failures occur before this activation (Niemann \& Wrenzycki 2000, Meirelles et al. 2004, Gad et al. 2012). Our results suggest adding vitamin $\mathrm{K}_{2}$ at the time of genome activation, a phase marked by an extended cell cycle and substantially increased demand for metabolic energy (Thompson et al. 1996, Tarazona et al. 2006), helps embryos complete this developmental step by increasing the mitochondrial activity.

As the treatment did not increase the number of hatched blastocysts, it appears that vitamin $\mathrm{K}_{2}$ did not stimulate developmental kinetics but rather rescued embryos that would otherwise have failed to develop. It has been proposed that development is affected by lower numbers of mitochondria or at least of copies of mtDNA (Ge et al. 2012). However, vitamin $K_{2}$ was shown not to have any impact on the mtDNA/nDNA ratio (Rao et al. 2009).

Embryo quality is evaluated routinely on the basis of morphological criteria in order to select embryos most

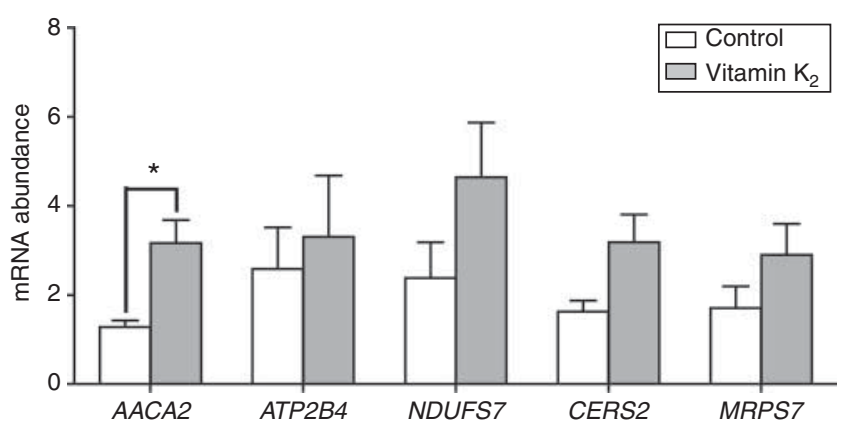

Figure 4 Validation of the differential expression of bovine embryo genes indicated by microarray analysis by quantitative RT-PCR: embryos were produced in vitro and cultured in a synthetic oviduct fluid without (white) or with vitamin $\mathrm{K}_{2}$ (gray). Values are expressed as mean \pm S.E.M. Quantification was normalized to endogenous $\beta$-actin transcript levels. *Significantly different $(P<0.05)$. 


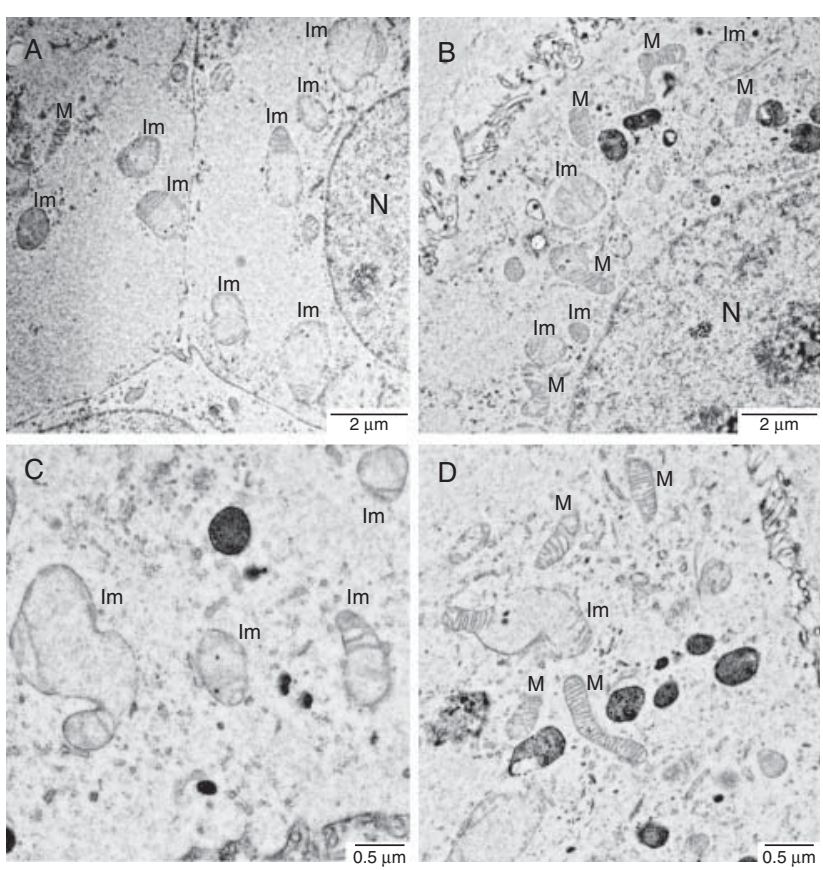

Figure 5 Electron micrographs showing morphological types of mitochondria in bovine embryos (blastomere stage) produced in vitro (A and C) embryo cultured in a synthetic oviduct fluid (SOF) without added vitamin $\mathrm{K}_{2}$, (B and D) embryo cultured in a SOF with added vitamin $\mathrm{K}_{2}$. The mitochondria of the control group have fewer cristae than those of the vitamin- $K_{2}$-treated group. $N$, nucleus; $M$, mature mitochondria; Im, immature mitochondria.

likely to survive cryopreservation (Hasler 2001, Abe et al. 2002a, Rizos et al. 2003) and produce gestation (Lindner \& Wright 1983, Hasler 2001, Abe et al. 2002b, Van Soom et al. 2003). Culture conditions are known to have a major impact on the quality of embryos (Abe et al. 2002a,b, Rizos et al. 2003, Van Soom et al. 2003). We observed that supplementation of the culture medium with vitamin $\mathrm{K}_{2}$ resulted in a high proportion of high-quality embryos.

Abe et al. (2002b) observed that the blastomeres of bovine embryos obtained in vivo and classified as 'excellent' quality contained a larger proportion of mature mitochondria. Similarly, we observed a higher yield of high-quality embryos and increased proportion of mature mitochondria in blastomeres, in association with vitamin $\mathrm{K}_{2}$ supplementation of the culture medium. Furthermore, the improved mitochondrial activity in the supplemented culture was most likely due to this increased proportion of mature mitochondria. The conditions that provide the signal for the maturation of the mitochondrial contingent are unknown. Mitochondria adopt their swollen immature shape with limited cristae during oocyte growth and keep a limited capacity to produce ATP until genome activation and thereafter acquire the mature shape (Mohr \& Trounson 1981, Plante \& King 1994). It has been reported in several studies that the developmental competence of mammalian oocytes and embryos is related to the state of maturity of the mitochondrial cohort (Shepard et al. 1998, Van Blerkom 2004, 2009, Crocco et al. 2011).

One reported manifestation of improved mitochondrial activity is increased $\beta$-oxidation of fatty acids, which generates ATP necessary for embryo development (Ferguson \& Leese 2006). Accordingly, the greater mitochondrial activity in the vitamin- $\mathrm{K}_{2}$ treated embryos coincided with increased expression (based on microarray analysis) of Acaa2, a gene known to play a role in $\beta$-oxidation of fatty acids in rat hepatocytes by regulating the generation of acetate (Yamashita et al. 2006). Embryos in this group also tended to show greater abundance of transcripts of CERS2. This gene is also involved in regulating mitochondrial function, as its ablation disrupts respiratory chain activity and leads to chronic oxidative stress and activation of stress-signaling pathways (PewznerJung et al. 2010, Zigdon et al. 2013). Consistent with these findings, embryos cultured in the presence of vitamin $\mathrm{K}_{2}$ also tended to exhibit heightened expression of NDUFS7 and MRPS7. Dysregulation of the former has been associated with impairment of the mitochondrial complex I of the electron transport chain (Triepels et al. 2001), while the latter gene has been associated with resistance to oxidative stress (Harding et al. 2003).

Based on transcriptome analysis, vitamin $\mathrm{K}_{2}$ supplementation was also found to have an impact on the transport of calcium, which plays a central role in the regulation of mitochondrial oxidative metabolism and is a co-factor of a major rate-limiting dehydrogenase of the TCA cycle (Duchen 2000, Dumollard et al. 2004). Impaired mitochondrial uptake of $\mathrm{Ca}^{2+}$ alters the spatiotemporal characteristics of cellular $\left[\mathrm{Ca}^{2+}\right]$ signaling and mitochondrial metabolism and might trigger pathological states that lead to cell death (Duchen 2000). Our findings might be related to differential expression of the ATP2B4 gene, the transcripts of which tended to be more abundant in the vitamin- $\mathrm{K}_{2}$-treated embryos. Several studies confirm that intracellular $\mathrm{Ca}^{2+}$ levels are related to the expression of ATP2B4 (Usachev et al. 2002, Schuh et al. 2004).

In conclusion, bovine embryos produced in vitro benefit significantly from vitamin $\mathrm{K}_{2}$ supplementation of the culture medium. This vitamin appears to improve embryonic phenotype when added at the onset of embryonic genome activation, a stage at which the initiation of mitochondrial maturation is also known to occur. The succeeding step in this research is to determine the potential of the resulting embryos to lead to healthy gestations that go full term.

\section{Declaration of interest}

The authors declare that there is no conflict of interest that could be perceived as prejudicing the impartiality of the research reported. 


\section{Funding}

This work was supported by NSERC Strategic Network EmbryoGENE NETPG 340825-06.

\section{Acknowledgements}

The authors thank Isabelle Laflamme and Alexandre Bastien (Université Laval, Canada) for technical assistance with, respectively, in vitro embryo procedures and confocal microscopy and image analysis.

\section{References}

Abe H, Yamashita S, Satoh T \& Hoshi H 2002a Accumulation of cytoplasmic lipid droplets in bovine embryos and cryotolerance of embryos developed in different culture systems using serum-free or serum-containing media. Molecular Reproduction and Development 61 57-66. (doi:10.1002/mrd.1131)

Abe H, Matsuzaki S \& Hoshi H 2002b Ultrastructural differences in bovine morulae classified as high and low qualities by morphological evaluation. Theriogenology 57 1273-1283. (doi:10.1016/ S0093-691X(02)00634-9)

Abramoff MD, Magalhaes PJ \& Ram SJ 2004 Image processing with ImageJ. Biophotonics International 11 36-42.

Bavister BD 1995 Culture of preimplantation embryos: facts and artifacts. Human Reproduction Update 1 91-148. (doi:10.1093/humupd/1.2.91)

Bermejo-Alvarez P, Rizos D, Rath D, Lonergan P \& Gutierrez-Adan A 2010 Sex determines the expression level of one third of the actively expressed genes in bovine blastocysts. PNAS 107 3394-3399. (doi:10.1073/pnas. 0913843107)

Crocco M, Alberio RH, Lauria L \& Mariano MI 2011 Effect of serum on the mitochondrial active area on developmental days 1 to 4 in in vitroproduced bovine embryos. Zygote 19 297-306. (doi:10.1017/ S0967199411000050)

Crocco MC, Kelmansky DM \& Mariano MI 2013 Does serum cause lipiddroplet accumulation in bovine embryos produced in vitro, during developmental days 1 to 4? Journal of Assisted Reproduction and Genetics 30 1377-1388. (doi:10.1007/s10815-013-0060-8)

Crosier AE, Farin PW, Dykstra MJ, Alexander JE \& Farin CE 2001 Ultrastructural morphometry of bovine blastocysts produced in vivo or in vitro. Biology of Reproduction 64 1375-1385. (doi:10.1095/ biolreprod64.5.1375)

Duchen MR 2000 Mitochondria and calcium: from cell signalling to cell death. Journal of Physiology 529 57-68. (doi:10.1111/j.1469-7793. 2000.00057.x)

Dumollard R, Marangos P, Fitzharris G, Swann K, Duchen M \& Carroll J 2004 Sperm-triggered $\left[\mathrm{Ca}^{2+}\right]$ oscillations and $\mathrm{Ca}^{2+}$ homeostasis in the mouse egg have an absolute requirement for mitochondrial ATP production. Development 131 3057-3067. (doi:10.1242/dev.01181)

Dumollard R, Carroll J, Duchen MR, Campbell K \& Swann K 2009 Mitochondrial function and redox state in mammalian embryos. Seminars in Cell \& Developmental Biology 20 346-353. (doi:10.1016/ j.semcdb.2008.12.013)

Fair T, Lonergan P, Dinnyes A, Cottell DC, Hyttel P, Ward FA \& Boland MP 2001 Ultrastructure of bovine blastocysts following cryopreservation: effect of method of blastocyst production. Molecular Reproduction and Development 58 186-195. (doi:10.1002/1098-2795(200102)58:2< 186::AID-MRD8 > 3.0.CO;2-N)

Ferguson EM \& Leese HJ 2006 A potential role for triglyceride as an energy source during bovine oocyte maturation and early embryo development. Molecular Reproduction and Development 73 1195-1201. (doi:10.1002/mrd.20494)

Gad A, Hoelker M, Besenfelder U, Havlicek V, Cinar U, Rings F, Held E, Dufort I, Sirard MA, Schellander K et al. 2012 Molecular mechanisms and pathways involved in bovine embryonic genome activation and their regulation by alternative in vivo and in vitro culture conditions. Biology of Reproduction 87 100. (doi:10.1095/biolreprod.112.099697)
Ge H, Tollner TL, Hu Z, Dai M, Li X, Guan H, Shan D, Zhang X, Lv J, Huang C et al. 2012 The importance of mitochondrial metabolic activity and mitochondrial DNA replication during oocyte maturation in vitro on oocyte quality and subsequent embryo developmental competence. Molecular Reproduction and Development 79 392-401. (doi:10.1002/mrd.22042)

Harding HP, Zhang Y, Zeng H, Novoa I, Lu PD, Calfon M, Sadri N, Yun C, Popko B, Paules R et al. 2003 An integrated stress response regulates amino acid metabolism and resistance to oxidative stress. Molecular Cell 11 619-633. (doi:10.1016/S1097-2765(03)00105-9)

Hasler JF 2001 Factors affecting frozen and fresh embryo transfer pregnancy rates in cattle. Theriogenology 56 1401-1415. (doi:10.1016/S0093691X(01)00643-4)

Lindner GM \& Wright RW Jr 1983 Bovine embryo morphology and evaluation. Theriogenology 20 407-416. (doi:10.1016/0093-691X (83)90201-7)

Meirelles FV, Caetano AR, Watanabe YF, Ripamonte P, Carambula SF, Merighe GK \& Garcia SM 2004 Genome activation and developmental block in bovine embryos. Animal Reproduction Science 82-83 13-20. (doi:10.1016/j.anireprosci.2004.05.012)

Mohr LR \& Trounson AO 1981 Structural changes associated with freezing of bovine embryos. Biology of Reproduction 25 1009-1025. (doi:10.1095/biolreprod25.5.1009)

Niemann H \& Wrenzycki C 2000 Alterations of expression of developmentally important genes in preimplantation bovine embryos by in vitro culture conditions: implications for subsequent development. Theriogenology 53 21-34. (doi:10.1016/S0093-691X(99)00237-X)

Olson SE \& Seidel GE Jr 2000 Culture of in vitro-produced bovine embryos with vitamin $\mathrm{E}$ improves development in vitro and after transfer to recipients. Biology of Reproduction 62 248-252. (doi:10.1095/biolreprod62.2.248)

Pewzner-Jung Y, Brenner O, Braun S, Laviad EL, Ben-Dor S, Feldmesser E, Horn-Saban S, Amann-Zalcenstein D, Raanan C, Berkutzki T et al. 2010 A critical role for ceramide synthase 2 in liver homeostasis: II. Insights into molecular changes leading to hepatopathy. Journal of Biological Chemistry 285 10911-10923. (doi:10.1074/jbc.M109.077610)

Phongnimitr T, Liang Y, Srirattana K, Panyawai K, Sripunya N, Treetampinich C \& Parnpai R 2013 Effect of L-carnitine on maturation, cryo-tolerance and embryo developmental competence of bovine oocytes. Animal Science Journal 84 719-725. (doi:10.1111/asj. 12067)

Plante L \& King WA 1994 Light and electron microscopic analysis of bovine embryos derived by in vitro and in vivo fertilization. Journal of Assisted Reproduction and Genetics 11 515-529. (doi:10.1007/BF02216032)

Plourde D, Vigneault C, Lemay A, Breton L, Gagne D, Laflamme I, Blondin P \& Robert C 2012 Contribution of oocyte source and culture conditions to phenotypic and transcriptomic variation in commercially produced bovine blastocysts. Theriogenology 116-131 e111-e113. (doi:10.1016/j.theriogenology.2012.01.027)

Poot M, Zhang YZ, Kramer JA, Wells KS, Jones LJ, Hanzel DK, Lugade AG, Singer VL \& Haugland RP 1996 Analysis of mitochondrial morphology and function with novel fixable fluorescent stains. Journal of Histochemistry and Cytochemistry 44 1363-1372. (doi:10.1177/44.12. 8985128)

Rao M, Li L, Demello C, Guo D, Jaber BL, Pereira BJ \& Balakrishnan VS 2009 Mitochondrial DNA injury and mortality in hemodialysis patients. Journal of the American Society of Nephrology 20 189-196. (doi:10.1681/ASN.2007091031)

Rizos D, Ward F, Duffy P, Boland MP \& Lonergan P 2002 Consequences of bovine oocyte maturation, fertilization or early embryo development in vitro versus in vivo: implications for blastocyst yield and blastocyst quality. Molecular Reproduction and Development 61 234-248. (doi:10.1002/mrd.1153)

Rizos D, Gutierrez-Adan A, Perez-Garnelo S, De La Fuente J, Boland MP \& Lonergan P 2003 Bovine embryo culture in the presence or absence of serum: implications for blastocyst development, cryotolerance, and messenger RNA expression. Biology of Reproduction 68 236-243. (doi:10.1095/biolreprod.102.007799)

Robert C, Nieminen J, Dufort I, Gagne D, Grant JR, Cagnone G, Plourde D, Nivet AL, Fournier E, Paquet E et al. 2011 Combining resources to obtain a comprehensive survey of the bovine embryo transcriptome through deep sequencing and microarrays. Molecular Reproduction and Development 78 651-664. (doi:10.1002/mrd.21364) 
Schmittgen TD \& Livak KJ 2008 Analyzing real-time PCR data by the comparative C(T) method. Nature Protocols 3 1101-1108. (doi:10.1038/ nprot.2008.73)

Schuh K, Cartwright EJ, Jankevics E, Bundschu K, Liebermann J, Williams JC, Armesilla AL, Emerson M, Oceandy D, Knobeloch KP et al. 2004 Plasma membrane $\mathrm{Ca}^{2+}$ ATPase 4 is required for sperm motility and male fertility. Journal of Biological Chemistry 279 28220-28226. (doi:10.1074/jbc.M312599200)

Shepard TH, Muffley LA \& Smith LT 1998 Ultrastructural study of mitochondria and their cristae in embryonic rats and primate (N. nemistrina). Anatomical Record 252 383-392. (doi:10.1002/ (SICI)1097-0185(199811)252:3<383::AID-AR6>3.0.CO;2-Z)

Sudano MJ, Mattos MCC, Fernandes CB, Mazieiro RR \& Landim-Alvarenga FC 2010 In vitro production of bovine embryos using Sigma antioxidant supplement ${ }^{\circledR}, \boldsymbol{\alpha}$-tocopherol and L-ascorbic acid. Animal Reproduction 7 42-48.

Takahashi T, Inaba Y, Somfai T, Kaneda M, Geshi M, Nagai T \& Manabe N 2013 Supplementation of culture medium with L-carnitine improves development and cryotolerance of bovine embryos produced in vitro. Reproduction, Fertility, and Development 25 589-599. (doi:10.1071/RD11262)

Tarazona AM, Rodriguez JI, Restrepo LF \& Olivera-Angel M 2006 Mitochondrial activity, distribution and segregation in bovine oocytes and in embryos produced in vitro. Reproduction in Domestic Animals 41 5-11. (doi:10.1111/j.1439-0531.2006.00615.x)

Thompson JG, Partridge RJ, Houghton FD, Cox CI \& Leese HJ 1996 Oxygen uptake and carbohydrate metabolism by in vitro derived bovine embryos. Journal of Reproduction and Fertility 106 299-306. (doi:10.1530/jrf.0. 1060299)

Thompson JG, McNaughton C, Gasparrini B, McGowan LT \& Tervit HR 2000 Effect of inhibitors and uncouplers of oxidative phosphorylation during compaction and blastulation of bovine embryos cultured in vitro. Journal of Reproduction and Fertility 118 47-55. (doi:10.1530/jrf.0. 1180047)

Triepels RH, Hanson BJ, van den Heuvel LP, Sundell L, Marusich MF, Smeitink JA \& Capaldi RA 2001 Human complex I defects can be resolved by monoclonal antibody analysis into distinct subunit assembly patterns. Journal of Biological Chemistry 276 8892-8897. (doi:10.1074/ jbc.M009903200)
Trimarchi JR, Liu L, Porterfield DM, Smith PJ \& Keefe DL 2000 Oxidative phosphorylation-dependent and -independent oxygen consumption by individual preimplantation mouse embryos. Biology of Reproduction 62 1866-1874. (doi:10.1095/biolreprod62.6.1866)

Usachev YM, DeMarco SJ, Campbell C, Strehler EE \& Thayer SA 2002 Bradykinin and ATP accelerate $\mathrm{Ca}(2+)$ efflux from rat sensory neurons via protein kinase $\mathrm{C}$ and the plasma membrane $\mathrm{Ca}(2+)$ pump isoform 4 . Neuron 33 113-122. (doi:10.1016/S0896-6273(01)00557-8)

Van Blerkom J 2004 Mitochondria in human oogenesis and preimplantation embryogenesis: engines of metabolism, ionic regulation and developmental competence. Reproduction 128 269-280. (doi:10.1530/rep.1.00240)

Van Blerkom J 2009 Mitochondria in early mammalian development. Seminars in Cell \& Developmental Biology 20 354-364. (doi:10.1016/ j.semcdb.2008.12.005)

Van Soom A, Mateusen B, Leroy J \& De Kruif A 2003 Assessment of mammalian embryo quality: what can we learn from embryo morphology? Reproductive Biomedicine Online 7 664-670. (doi:10. 1016/S1472-6483(10)62089-5)

Vos M, Esposito G, Edirisinghe JN, Vilain S, Haddad DM, Slabbaert JR, Van Meensel S, Schaap O, De Strooper B, Meganathan R et al. 2012 Vitamin $\mathrm{K} 2$ is a mitochondrial electron carrier that rescues pink1 deficiency. Science 336 1306-1310. (doi:10.1126/science.1218632)

Yamashita H, Itsuki A, Kimoto M, Hiemori M \& Tsuji H 2006 Acetate generation in rat liver mitochondria; acetyl-CoA hydrolase activity is demonstrated by 3-ketoacyl-CoA thiolase. Biochimica et Biophysica Acta 1761 17-23. (doi:10.1016/j.bbalip.2006.01.001)

Zigdon H, Kogot-Levin A, Park JW, Goldschmidt R, Kelly S, Merrill AH Jr, Scherz A, Pewzner-Jung Y, Saada A \& Futerman AH 2013 Ablation of ceramide synthase 2 causes chronic oxidative stress due to disruption of the mitochondrial respiratory chain. Journal of Biological Chemistry $\mathbf{2 8 8}$ 4947-4956. (doi:10.1074/jbc.M112.402719)

Received 23 June 2014

First decision 24 July 2014

Accepted 8 August 2014 